\title{
LOS 7 PRINCIPIOS DE GESTIÓN DE LA CALIDAD EN ISO 9001
}

\section{SEVEN PRINCIPLES OF QUALITY MANAGEMENT IN ISO 9001}

Sandra Sirvent Asensi ${ }^{1}$

Victor Gisbert Soler ${ }^{2}$

Elena Pérez Bernabeu ${ }^{3}$

1. Graduada en Diseño Industrial y Desarrollo del Producto. Máster Universitario en Organización y Logística. Universidad Politécnica de Valencia - Campus de Alcoy (España). E-mail: sansiras@alumni.upv.es

2. Doctor Ingeniero Industrial. Departamento de Estadística e Investigación Operativa Aplicadas y Calidad. Universidad Politécnica de Valencia (España). E-mail: vgisber@eio.upv.es

3. Doctor Ingeniero en Organización Industrial. Departamento de Estadística e Investigación Operativa Aplicadas y Calidad. Universidad Politécnica de Valencia (España).E-mail: elpeber@eio.upv.es

\section{Citación sugerida:}

Sirvent Asensi, S., Gisbert Soler, V. y Pérez Bernabeu, E. (2017). Los 7 principios de gestión de la calidad en ISO 9001. 3C Empresa: investigación y pensamiento crítico, Edición Especial, 10-18. DOI: <http://dx.doi.org/10.17993/3cemp.2017.especial.10-18/>. 


\section{RESUMEN}

Tras la última revisión de la familia de normas ISO 9000, y más en concreto, la ISO 9001, con versión del 2015, los principios de gestión de la calidad sufrieron algún cambio. Por ejemplo, la desaparición del enfoque de sistema para la gestión o, el cambio de nombre de algunos de los principios los cuales abarcan un campo más amplio que en la versión anterior. En este artículo vamos a tratar de hacer una comparación entre las dos versiones, indicar algún ejemplo e integrar el Lean Manufacturing en el campo de la calidad.

\section{ABSTRACT}

After the last revision of the ISO 9000 standards, and more precisely, ISO 9001, version 2015 , the principles of quality management suffered some changes. For instance, some of the mentioned principles changed their name and cover a wider field than previous version. In this article, we will make a comparison between the 2 versions, explain some example and include the Lean Manufacturing in quality field.

\section{PALABRAS CLAVE}

Calidad, Lean, Principios, ISO 9001, Gestión.

\section{KEY WORDS}

Quality, Lean, Principles, ISO 9001, Management. 


\section{INTRODUCCIÓN}

De todos es sabido que ISO 9001, Sistema de Gestión de la Calidad, constituye uno de los sistemas de gestión más implantados hoy día en las organizaciones globalmente hablando. Esta familia de normas estandariza los sistemas de gestión de la calidad, proporcionando una base consolidada de actuación en relación con la gestión de la calidad a nivel internacional, con las consabidas ventajas para las organizaciones que deciden su aplicación.

En concreto, la ISO 9001, es la norma referencia que usan las organizaciones para diseñar e implementar su sistema de gestión de la calidad. La norma recoge los requisitos del sistema y está prevista para uso contractual, reglamentario o en certificación.

\section{1 ¿QUÉ ES LA GESTIÓN DE LA CALIDAD?}

La gestión de la calidad es el proceso de cambio que ha sufrido el concepto de calidad y sus implicaciones. Para entender la gestión de la calidad, previamente, debemos hablar de la calidad como control de calidad y del proceso de evolución a gestión de la calidad total.

En primer lugar, pasamos de una etapa donde la calidad solo se refiere al control final de los productos, separándolos en buenos o malos. A continuación, se pasa a la etapa de control de calidad del proceso en la que el lema principal es que "la calidad no se controla, sino que se fabrica". Por lo tanto, el control de calidad se inició con la idea de la inspección, y, para garantizar esta calidad se precisa de la participación de todos los miembros de la organización.

La siguiente etapa a la que se llega es la de una calidad de diseño que, aparte de corregir o reducir los defectos, también los previene.

Por lo tanto, el camino hacia una calidad total significa crear una nueva cultura, mantener un liderazgo, desarrollar al personal y el trabajo en equipo, desarrollar a los proveedores, tener un enfoque hacia el cliente y planificar la calidad.

Y, así, es como llegamos al concepto de gestión de la calidad, es decir, es la evolución de la calidad a calidad total, por la cual entendemos que comprende todos los aspectos de la organización e involucra a todos sus miembros.

Dado el contexto socio-económico actual, en el que existe un proceso de cambio acelerado y la competitividad es global, las organizaciones luchan por sobrevivir y destacar logrando una mayor eficiencia y brindando productos y servicios de calidad. Por eso, muchas empresas reconocen la importancia de la calidad y su gestión a la hora de lograr destacar entre la competencia teniendo una metodología que les sirva de soporte.

A continuación, se muestra una tabla comparativa entre el viejo enfoque (control de calidad) y el nuevo (gestión de la calidad total) ${ }^{3}$. 
Cumplir los estándares y procedimientos Satisfacer las expectativas del cliente. definidos.

Invertir tiempo y dinero para conseguir Ahorrar tiempo y dinero haciendo las cosas con mejorar la calidad. calidad.

La calidad es responsabilidad de unos pocos.

Detectar los errores producidos en el proceso.

La calidad es responsabilidad de todos.

Evitar los errores, haciendo las cosas bien la primera vez.

Tabla 1. Comparativa entre viejo enfoque y nuevo enfoque.

\section{LOS 7 PRINCIPIOS DE GESTIÓN DE LA CALIDAD}

La familia de normas ISO 9000 se basa en siete principios básicos de gestión de la calidad. Anteriormente, eran ocho, pero con la nueva revisión del 2015, los principios han quedado en siete. A continuación, vamos a ver más detalladamente cada uno de ellos.

\section{Enfoque al cliente}

"Las organizaciones dependen de sus clientes por lo que deberían comprender sus necesidades actuales y futuras, satisfacer sus requisitos y esforzarse en exceder sus expectativas" 7 . Es por eso que se le da prioridad a este principio, ya que el cliente representa ganancias y más trabajo para las organizaciones. Además, la disponibilidad de recursos centrados en cumplir con la satisfacción del cliente produce un alto grado de eficiencia, lo cual representa una ventaja para la organización y una fidelización del cliente 4 .

Un ejemplo de enfoque al cliente incluido en la política de calidad sería:

"Deseamos lograr la máxima satisfacción de nuestros clientes valorando sus necesidades y expectativas y teniéndolas en cuenta en el establecimiento de los objetivos y estrategias."

\section{Liderazgo}

"Los líderes establecen la unidad de propósito y la orientación de la organización. Ellos deberían crear y mantener un ambiente interno, en el cual el personal pueda llegar a involucrarse totalmente en el logro de los objetivos de la organización" ${ }^{7}$. Es por eso que las organizaciones necesitan líderes que muevan masas, es decir, que sus ideologías deben traer beneficios para todos. Entre las ventajas para la organización podemos destacar la implicación y estimulación de la participación del personal, ofrecer a la toda la organización la máxima información y dejar clara la visión de futuro para toda la organización ${ }^{4}$.

Algunas de las frases que un buen líder no debe olvidar nunca son:

- Las 6 palabras más importantes: Yo acepto que cometí un error.

- Las 5 palabras más importantes: Tú hiciste un buen trabajo

- Las 4 palabras más importantes: ¿Cuál es tu opinión?

- Las tres palabras más importantes: ¿Pudieras por favor? 
- Las dos palabras más importantes: Muchas gracias

- La palabra más importante: Nosotros

- La palabra menos importante: Yo

\section{Compromiso de las personas}

El compromiso de las personas que están involucradas en una organización es vital, ya que éstas son la parte más importante y posibilita que sus habilidades sean usadas para el beneficio de la organización.

La participación del personal es básica para mantener en funcionamiento un sistema de gestión de calidad. Además, es del personal operario del que se pueden extraer las mejores ideas, ya que estos son los que pasan parte de su día a día con el producto o servicio que la organización ofrece.

Las ventajas para la organización son la motivación, compromiso y toma de conciencia del papel de cada uno de los clientes internos (o empleados) de la organización. Además, existirán corrientes de opinión favorables a la participación activa en las actividades de mejora continua (lean manufacturing) ${ }^{3}$.

Un ejemplo de compromiso de las personas podría ser la formación, competencias y experiencia que el operario puede demostrar a la empresa para desempeñar a la perfección un determinado trabajo.

\section{Enfoque a procesos}

"Un resultado deseado se alcanza más eficientemente cuando las actividades y los recursos relacionados se gestionan como un proceso" ${ }^{7}$. Por eso, la organización debe estructurarse mediante procesos y marcar objetivos para cada uno de ellos. Para llevar a cabo un buen control, las grandes empresas deberían subdividirse en varios procesos, lo cual les facilitará una mejor organización global de la misma.

Una buena gestión de los equipos, instalaciones e infraestructuras nos ayuda a analizar los costes y eliminar aquellos que sean inútiles, como, por ejemplo, disminuyendo los tiempos de máquinas o alargando el ciclo de vida de los equipos. Además, con una buena planificación, se alcanzan mejor los resultados.

El sistema de gestión basado en los procesos y la mejora continua orientada directamente hacia la identificación de las oportunidades de mejora ${ }^{5}$.

Un ejemplo de enfoque basado en procesos sería una empresa cuya función es la producción de un producto y su posterior venta, pero, además, tienen un sistema de soporte, el proceso de producción está dividido por actividades y se intentan minimizar los costes inútiles. 
Mejora

"La mejora continua del desempeño global de la organización debe ser un objetivo permanente" ${ }^{\prime \prime}$. Se trata de algo intangible que la organización debe comprender para poder darle valor agregado, es decir, mejorar de forma interminable, sin estancarse.

Podemos encontrar dos tipos de mejora, la primera, mediante un avance tecnológico y, la segunda, mediante la mejora de todo el proceso productivo. Alcanzar los mejores resultados no es labor de un día, sino que se trata de proceso progresivo en el que no puede haber retrocesos.

Sin mejora continua no se puede garantizar un nivel de calidad ni tomar decisiones acertadas ni cumplir las metas y objetivos.

Con la mejora continua, las organizaciones pueden situarse en primera línea de competencia ${ }^{3}$.

Algunos ejemplos de mejora continua son la inclusión de la filosofía Kaizen dentro de la organización, la utilización de metodologías de mejora como pueden ser los talleres HOSHIN o SMED, Six-Sigma o la utilización de paneles de gestión visual en la empresa para que todo el personal pueda ver los progresos de ésta y se sientan partícipes.

\section{Toma de decisiones basadas en la evidencia}

"Las decisiones deben basarse, en la medida de lo posible, en el análisis de datos y a partir de la mejor información"7. Toda decisión que impacte a la calidad del producto debe ser tomada ante un hecho previo que garantice o reduzca la posibilidad de un error.

Las ventajas para la organización son que la toma de decisiones basada en informaciones veraces y evidenciables la conducirá por el buen camino de la calidad. Además, demuestra que las posibilidades y oportunidades existentes son canalizadas hacia su realización de forma eficaz.

Un ejemplo de toma de decisiones basadas en la evidencia puede ser una organización la cual tenga limitaciones en sus recursos, pero éstos los utiliza de forma más analítica convirtiendo los datos en información y usándola para generar y cumplir nuevos objetivos.

\section{Gestión de las relaciones}

La organización es interdependiente de sus clientes y proveedores, por lo que una relación mutuamente beneficiosa aumenta la capacidad de todos para crear valor. El cliente no se conforma con que una organización esté certificada, sino que requiere que los proveedores también cumplan con esta certificación, lo cual indica que la materia prima con la que se realiza el producto o servicio final es de calidad y cumple con los requisitos del cliente directo.

La ventaja para la organización es que fomenta la creación de valor añadido, provoca un entendimiento claro de las necesidades y expectativas del cliente y consigue una reducción de tiempos, costes y recursos junto a un aumento de la rentabilidad en los resultados ${ }^{3}$. 
Por ejemplo, una organización cuyas relaciones con el proveedor y el cliente son buenas, propiciará que el cliente esté satisfecho y que la organización sólo se tenga que preocupar de mantener estas relaciones.

En resumen, el uso de estos siete principios de gestión para una organización dará como resultado beneficios para todas las partes interesadas, así como, una mejora en la rentabilidad, la creación de valor y el incremento de la estabilidad.

\section{COMPARACIÓN ENTRE ISO 9001_2008 E ISO 9001:2015}

Como ya hemos dicho anteriormente, la nueva versión de ISO 9001:2015 ha supuesto la eliminación de uno de los ocho principios de calidad, los cuales han quedado en siete. Éste se trata del enfoque de sistema para la gestión, pero lo podemos encontrar implícito en los otros siete.

Hay que mencionar que en la norma anterior, estos principios de gestión de la calidad no aparecían pero, en la versión actual, todos estos principios están descritos en la Norma ISO 9000:2015 y en cada uno de ellos aparece una declaración, una base razonada, es decir, una explicación de porqué ese principio es importante para la organización, los beneficios asociados a su aplicación y las acciones posibles a desarrollar para aplicar cada principio concreto y mejorar el desempeño de la organización.

A continuación vamos a mostrar una tabla comparativa de los principios de gestión entre las dos versiones de ISO 9001, es decir, la actual, ISO 9001:2015 y su predecesora, la ISO 9001:2018

ISO 9001:2008

ISO 9001:2015

\begin{tabular}{|c|c|}
\hline 1. Enfoque al cliente. & 1. Enfoque al cliente. \\
\hline 2. Liderazgo. & 2. Liderazgo. \\
\hline 3. Participación del personal. & 3. Compromiso de las personas. \\
\hline 4. Enfoque basado en procesos. & 4. Enfoque a procesos. \\
\hline \multicolumn{2}{|l|}{ 5. Enfoque de sistema para la gestión. } \\
\hline 6. Mejora continua. & 5. Mejora. \\
\hline $\begin{array}{l}\text { 7. Enfoque basado en hechos para la toma de } \\
\text { decisión. }\end{array}$ & 6. Toma de decisiones basadas en la evidencia. \\
\hline $\begin{array}{l}\text { 8. Relaciones mutuamente beneficiosas con el } \\
\text { proveedor. }\end{array}$ & 7. Gestión de las relaciones. \\
\hline
\end{tabular}

Tabla 2. Comparativa de los principios de gestión entre la ISO 9001:2015 y la ISO 9001:2018. 


\section{CONCLUSIONES}

En definitiva, dentro de los siete principios de gestión de la calidad hay que considerar uno como el más importante el cual está relacionado con los demás, y, este es, el enfoque al cliente. Sin éste, ninguna organización existiría ya que el cliente es el que siempre tiene la razón y los productos o servicios se realizan pensando en él.

Hay que mencionar que otro de los principios que también es considerado de los más importantes, es el del compromiso de las personas, ya que, al igual que el anterior, se trata de clientes internos de la empresa y que, sin ellos, tampoco existiría la organización. Con un buen compromiso de las personas y haciéndoles partícipes de los objetivos de ésta, se verá reflejado en el mantenimiento de la gestión de la calidad.

Con el compromiso de las personas, la organización puede llevar a cabo más fácilmente la instauración de la mejora continua, lo cual reafirma el mantenimiento de la gestión de la calidad mencionado anteriormente.

Si siguiéramos haciendo una breve conclusión de todos los principios, al final, llegaríamos a la misma conclusión, es decir, que todos los principios están relacionados con un nexo en común: el cliente. 


\section{REFERENCIAS BIBLIOGRÁFICAS}

Libros:

- (1) Miró Sacanelles, María y García de Mateo, Ma Ángeles. Principios básicos de la calidad: Normalización y Certificación. Ediciones Roble, septiembre 2012.

Sitios web:

- (2) Apcer Group. 7 principios de gestión de calidad, según ISO 9001 [en línea]. [Consulta: 20 de abril de 2017]. Disponible en: http://apcergroup.com/espana/index.php/es/newsroom/775/7-principios-degestion-de-calidad-segun-la-iso-9001

Blog:

- (3) Anónimo. "7 principios de gestión de la calidad". Sistemas y calidad total [blog]. 18 de junio de 2016. [Consulta: 20 de abril de 2017]. Disponible en: http://www.sistemasycalidadtotal.com/calidad-total/los-7-principios-degestion-de-la-calidad/

- (4) Anónimo. "ISO 9001 Principios de los Sistemas de Gestión de la Calidad". Nueva ISO 9001:2015 [blog]. 13 de octubre de 2014. [Consulta: 20 de abril de 2017]. Disponible en: http://www.nueva-iso-9001-2015.com/2014/10/iso-9001principios-sistemas-gestion-calidad/

- (5) Anónimo. "Los 7 principios de gestión de la calidad según ISO 9001:2015". Qualired [blog]. 3 de diciembre de 2015. [Consulta: 20 de abril de 2017]. Disponible en: http://www.qualired.com/despachos1.asp?cod des=62662

- (6). Jiménez, Daniel. "Los 7 principios de gestión de calidad - DIS/ISO 9001:2015". Pymes y calidad 2.0 [blog]. 11 de octubre de 2014. [Consulta: 20 de abril de 2017]. Disponible en: http://www.pymesycalidad20.com/los-7-principios-de-lagestion-de-calidad-disiso-90012015.html

Normas:

- (7) UNE-EN ISO 9001:2015. Sistemas de gestión de calidad. Requisitos.

- (8) UNE-EN ISO 9001:2008. Sistemas de gestión de calidad. Requisitos. 